\title{
Risperidone was effective for aggression in adolescents with disruptive behaviour disorders and below average intelligence
}

Buitelaar JK, van der Gaag RJ, Cohen-Kettenis P, et al. A randomized controlled trial of risperidone in the treatment of
aggression in hospitalized adolescents with subaverage cognitive abilities. J Clin Psychiatry 2001 Apr;62:239-48.

QUESTION: In hospitalised young adolescents with disruptive behaviour disorders and below average intelligence, is risperidone effective and safe for treating aggression?

\section{Design}

Randomised \{allocation concealed*\}†, blinded (clinicians, teachers, patients, psychologists, laboratory personnel, and trial monitor $\} \dagger^{*}$, placebo controlled trial with 6 weeks of follow up.

\section{Setting}

2 tertiary referral centres in the Netherlands.

\section{Patients}

38 patients who were 12-18 years of age (mean age 14 y, $87 \%$ boys), had overt aggressive behaviour persisting during hospitalisation (score $\geqslant 1$ on the modified Overt Aggression Scale [OAS-M] rated by nurses); aggressive behaviour not responding to behavioural treatment; clinical indication for study drug; a principal DSM-IV diagnosis of conduct disorder, oppositional defiant disorder or attention deficit hyperactivity disorder; and a Full Scale Intelligence Quotient of $60-90$ on the Wechsler Intelligence Scale for Children-Revised. Exclusion criteria included neurological, cardiac, pulmonary, or hepatic diseases; primary mood disorders; schizophrenia or other active psychoses; suicidal tendencies; comorbid substance abuse disorder; pregnancy or inadequate contraception; major change in treatment strategy expected in near future; and inability to discontinue current psychotropic medication. $95 \%$ of patients completed the study at 6 weeks.

\section{Intervention}

Patients were allocated to risperidone $(\mathrm{n}=19)$ or placebo $(\mathrm{n}=19)$. Study drug titration started from $0.5 \mathrm{mg}$ twice daily to a maximum of $5 \mathrm{mg}$ twice daily.

\section{Main outcome measures}

Severity of symptoms (Clinical Global ImpressionSeverity of Illness scale [CGI-S]). Secondary outcomes were the OAS-M, Aberrant Behavior Checklist (ABC), and Extrapyramidal Symptom Rating Scale (ESRS).

\section{Main results}

Analysis was by intention to treat. Fewer patients in the risperidone group than the placebo group had "markedly" to "extremely severely disturbed" CGI-S scores (score $5-7)(\mathrm{p}<0.001)$ at 6 weeks (table). The risperidone group had greater improvement in ABC scores rated by teachers than did the placebo group (mean score change from baseline $14.9 v 3.7, \mathrm{p}<0.05$ ). The difference between groups for change from baseline was not statistically significant for nurse rated overall $\mathrm{ABC}$ scores, overall OAS-M scores, or any subscale of ESRS except for parkinsonism, which was increased in the risperidone group but not in the placebo group (mean score change $0.6 v-0.5, \mathrm{p}<0.05)$.

\section{Conclusion}

In hospitalised young adolescents with disruptive behaviour disorders and subaverage intelligence, risperidone was safe and more effective than placebo for treating aggression.

*See glossary.

$\dagger$ Information provided by author.
Source of funding: Janssen-Cilag BV the Netherlands.

For correspondence: $D$ JK Buitelaar,

Department of Child Psychiatry, $P$ O Box $85500,3508 \mathrm{GA}$

Utrecht, The

Netherlands. Fax +31

302542286.
Risperidone v placebo for aggression in adolescents with disruptive behaviour disorders and below average intelligence

Outcome at 6 weeks $\quad$ Risperidone Placebo RRR $(95 \%$ CI) NNT (CI)

Markedly to extremely disturbed§ $\quad 21 \% \quad 84 \% \quad 75 \%$ (46 to 90$) \quad 2$ (2 to 4$)$

$\neq$ Abbreviations defined in glossary; RRR, NNT, and $\mathrm{Cl}$ calculated from data in article.

$\S$ Score of 5-7 on Clinical Global Impression-Severity of Illness Scale.

\section{COMMENTARY}

The study by Buitelaar $e t$ al answers a range of questions, but is particularly useful for 3 reasons. Firstly, it studies the effect of risperidone on aggressive behaviours in a group for which this is the primary symptom, and the aggression is less contaminated by the presence of other symptoms, such as those in patients with a pervasive developmental disorder or a thought disorder. Secondly, it focuses on admitted to hospital aggressive young adolescents with disruptive behaviour disorders, who typically require pharmacological treatment because they have been unmanageable as outpatients with conventional treatments and are admitted to control their aggression. Moreover, in most hospitals, length of stay and insurance requirements indicate that patients must be discharged rapidly, so behavioural therapies are unlikely to be rapid enough when used alone. Thirdly, it focuses on individuals with "subaverage intelligence", a group that has not been specifically studied previously but is likely to have more limited alternative adaptive capacities - leaving them more likely to be overtly aggressive.

Some caution is necessary in the interpretation of scores at the end of the washout period ("washout effect") because they are confounded by withdrawal effects, not just the absence of the drug treatment. This caveat remains important in spite of the lack of dyskinesias evident at washout on the ESRS. A virtue of the study is that the doses were not high, as too often occurs clinically.

The results complement an earlier study of younger outpatients with conduct disorder, ${ }^{1}$ which also indicated the beneficial effects of risperidone for aggressive youth. More research will give increasing specificity to our knowledge about this topic. For example, it will be important to compare the effects of risperidone with other medications and behavioural treatments for aggression and to carefully examine dose effects.

J Gerald Young, MD New York University Medical Center New York, New York, USA

1 Findling RL, McNamara NK, Branicky LA, et al. A double-blind pilot study of risperidone in the treatment of conduct disorder. J Am Acad Child Adolesc Psychiatry 2000;39:509-16. 